\title{
Evaluation of Medical Officers' Certificate Programme (MOCP) Course Competency Based Learning
}

\author{
S. Saunik ${ }^{1}$, M. Phadke², R. Nair ${ }^{3}$, P. Jakkal ${ }^{4}$, P. Menon ${ }^{5}$, A. Patil ${ }^{6}$, I. Kundan ${ }^{7}$ \\ ${ }^{1}$ Public Health Department, Mantralaya, Mumbai, India \\ ${ }^{2}$ Sr. Adv. NHM, UNICEF, Mumbai, India \\ ${ }^{3}$ Nutrition Specialist, UNICEF, Mumbai, India \\ ${ }^{4}$ National Health Mission, Mumbai, India \\ ${ }^{5}$ Department of Genetics, Immunology, Biochemistry and Nutrition Maharashta University of Health Sciences, Pune \\ Center, India \\ ${ }^{6}$ Additional Director of Health Sciences, State Family Welfare Bureau, Pune, India \\ ${ }^{7}$ M.D. National Health Mission, Mumbai, India
}

\section{Article Info}

Article history:

Received Oct 17, 2015

Revised Nov 20, 2015

Accepted Nov 26, 2015

\section{Keyword:}

MOCP

Evaluation

India

\begin{abstract}
Medical Officers' Certificate Programme (MOCP) is a 6 months training programme in Pediatrics/Medicine at Medical colleges wherein doctors work like postgraduate students, learn various OPD, IPD, (Out Patients and In Patient Department) procedures, attain hands on skills, perform day and night duties, attend postgraduate training programmes and specialty clinics. This is a course unique to Maharashtra. It has been designed to overcome shortage of Pediatrians \& Physicians in the state. Public Health Dept deputed 28 Medical Officers of Primary Health Centers to various Medical Colleges. At the end of 6 months training course they were evaluated during 2012-2013.It was done by questionnaire used before and after training. It was observed that OPD increased by $24 \%$ and IPD by $54 \%$. There was a decrease in the number of cases referred to tertiary centers by $24 \%$, post MOCP training. Infant immunization increased by $35 \%$ after training. Number of children with severe acute malnutrition/moderate acute malnutrition treated increased by $22 \%$, neonatal emergencies, resuscitation, sepsis, jaundice patients treated, increased by $36 \%$. No of adults with diarrhoea and snake bite treated increased by $40 \%$ \& $63 \%$ respectively. No. of ECGs taken and myocardial infarctions managed also has shown rising trend. Thus, there was tremendous benefit to the patients after MOCP training. Skill of doctors was found to have enhanced. It is therefore recommended that such novel trainings should be imparted in other states of India too.
\end{abstract}

Copyright (c) 2015 Institute of Advanced Engineering and Science. All rights reserved.

Corresponding Author:

M. Phadke

UNICEF, Mumbai, India

Email:drmapaa@yahoo.com

\section{INTRODUCTION}

Public Health Dept. of Govt. of Maharashtra started two unique teaching programmes for medical officers- MOCP i.e. Medical Officers' Certificate Programme and CCCN i.e. Certificate Course in Clinical Nutrition. MOCP is a short term 6 months comprehensive training programme for Medical Officers from primary Health Centers to work in the Dept. of Pediatrics/Medicine of Medical Colleges in the State of Maharashtra. Medical Officers are taught like residents and postgraduate students, working in OPD, IPD, attending day and night duties, specialty clinics, national programmes specially related to nutrition, emergency treatments, skill building through various procedures like IV lines rescuscitation, intubation, ECG, lumber punctures etc. They are given interactive power point, Video Conferencing lectures by eminent specialists like Professors, Clinicians, Padma awardees on relevant subjects, ethics \& motivation. Examination is taken at the end of 6 months. This course is expected to overcome to some extent, vacancy 
and short fall of Pediatricians \& Physicians at the Primary Health Centers for the Public Health Dept. of Maharashtra.

Through the present paper, we have analyzed \& evaluated the efficacy of MOCP courses imparted to Medical Officers. Evaluation has been done to find out if the clinical skills of the medical officers have improved or otherwise and if they have achieved expected level of competence.

\section{MATERIAL AND METHODS}

Medical Officers trained in 2012-2013 were selected for the present study. They were trained in Medical Colleges and had passed the theory and practical examination. They were reposted back to the Original primary health centers where they were posted earlier. Data was collected from Medical Officers pre training i.e. from Feb. to May 2012 and post training period from Feb to May 2013. Questionnaire was sent to 56 Medical officers from the second batch of training, 32 Medical Officers replied out of whom 28 had completed the questionnaire. So, they formed the study group. Structured questionnaire included questions on the number of OPD \& IPD case pre and post MOCP training, difference in case referrals, difference in neonatal emergency management, SAM (Severe Acute Malnutrition) management, medical emergencies before and after training.

\section{OBSERVATIONS}

\subsection{Background Characteristics}

A sample of 28 MOCP trained Medical Officers was studied for the present report. These Medical Officers belonged to different districts of Maharashtra. They are Jalna - 1, Latur - 4, Wardha - 3, Nandurbar 1 , Aurangabad - 2 , Nasik - 3 , Bhandara - 1, Parbhani - 1 , Raigad - 1, Gondia - 1, Gadchiroli - 1 , Nanded - 1, Pune - 1 , Nagpur - 1 , Jalgaon - 1 , Thane - 1 , Satara - 2, Washim - 1. Medical Officers were provided training in Medicine and Paediatric departments in 9 Medical colleges of Maharashtra. Out of these 28 Medical Officers, 14 received training in Paediatrics and remaining 14 received training in Medicine.

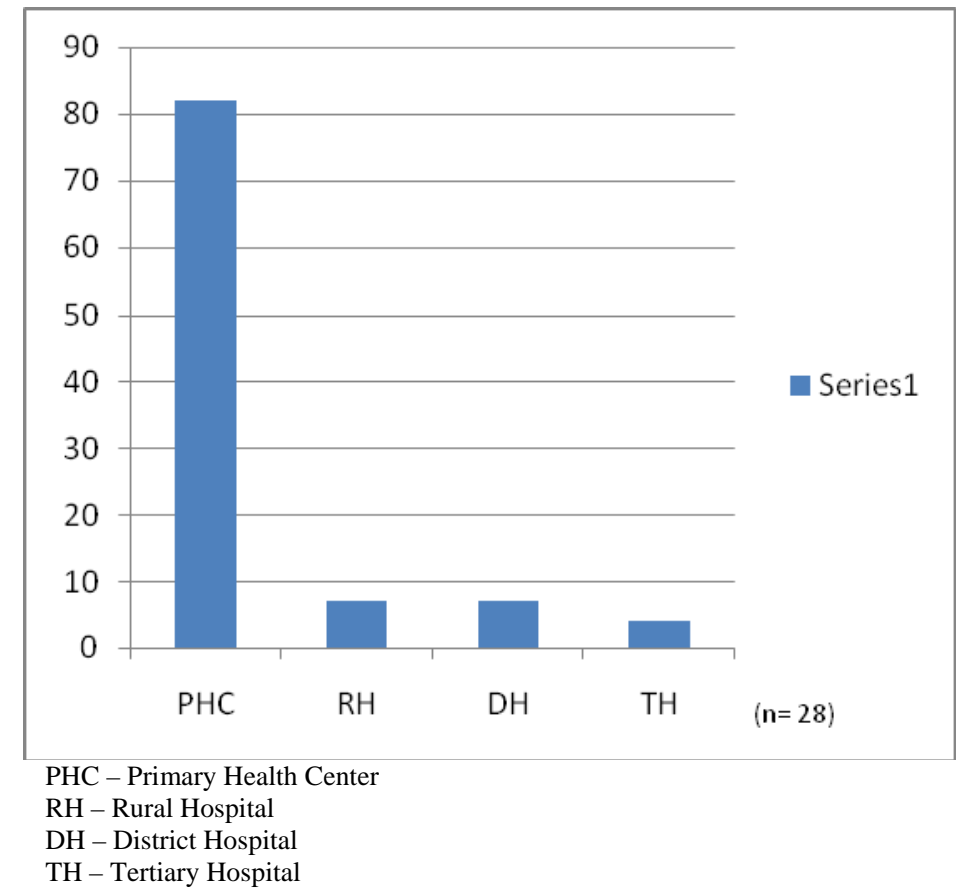

Figure 1. Area wise distribution of posting of MOCP trained Medical Officers

From the diagram in Figure 1 it can be seen that out of total 28 trained Medical Officers from the sample, majority i.e. $82 \%$ belonged to PHC followed by 7\% each from RH and DH and 4\% from $\mathrm{TH}$. 


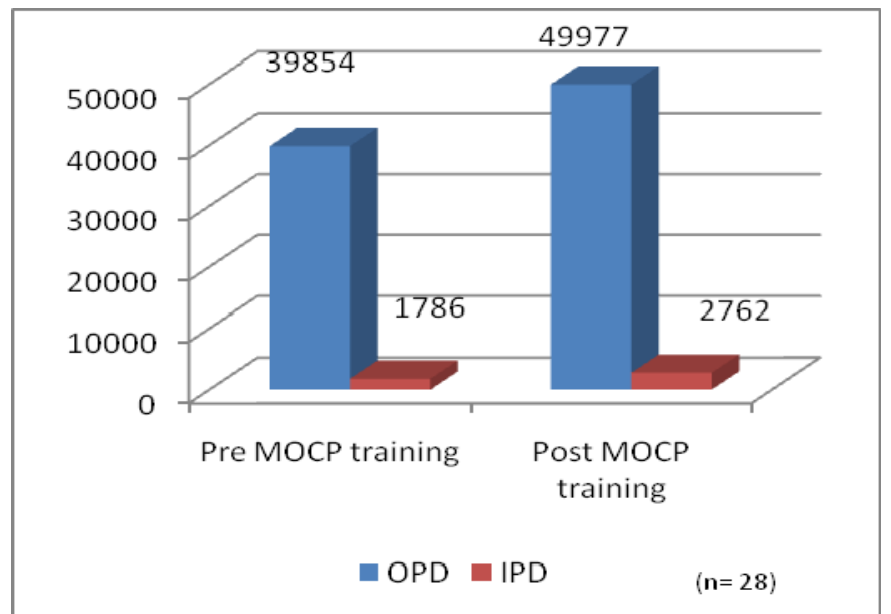

Figure 1. Number of OPD and IPD patients pre and post MOCP training

Figure 2 shows the number of OPD and IPD patients pre and post MOCP training. From the figure it can be seen that there has been an increase in the number of both OPD and IPD patients by 25\% and 54\% respectively in post MOCP training than pre MOCP training.

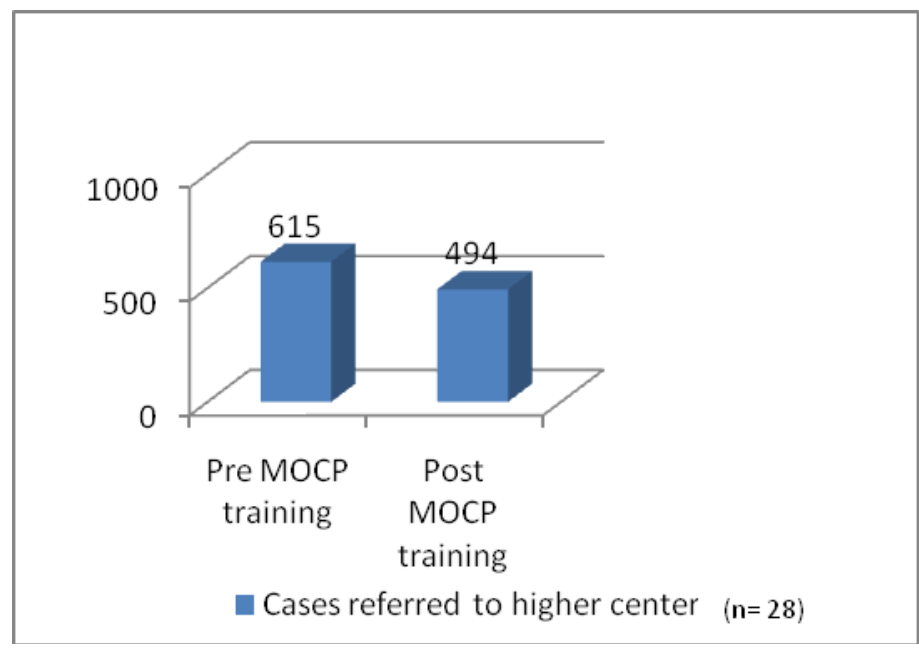

Figure 2. Number of cases referred to higher centers pre and post MOCP training

Figure 3 shows the number of referrals before and after training. From the figure it can be seen that there has been decrease in the number of cases referred to higher tertiary centers in post MOCP training by $24 \%$ than pre MOCP training. It shows that after receiving MOCP training 24\% of cases have been managed by the MOCP trained Medical Officers which were earlier referred to higher centers.

Figure 4 shows the number of patients managed in paediatric age group pre and post MOCP training. From the figure it can be seen that there has been a rise in all the indicators of paediatric management. Infant immunization has increased by 35\%, number of SAM/MAM treated has increased by $22 \%$, newborn resuscitation has increased by around $190 \%$ and treatment of neonatal emergencies like sepsis, jaundice, etc has increased by $36 \%$ in post MOCP training than pre MOCP training.

Figure 5 shows the management of number of patients suffering from diarrheoa, myocardial infarction and coronary artery disease, snakebite, in pre and post MOCP training. From the figure it can be seen that there has been a rise in all the three diseases management. Number of diarrheal patients managed has increased by $40 \%$, number of myocardial infarction and coronary artery disease managed has drastically increased and snakebite management has increased by 63\% in post MOCP training than pre MOCP training. 


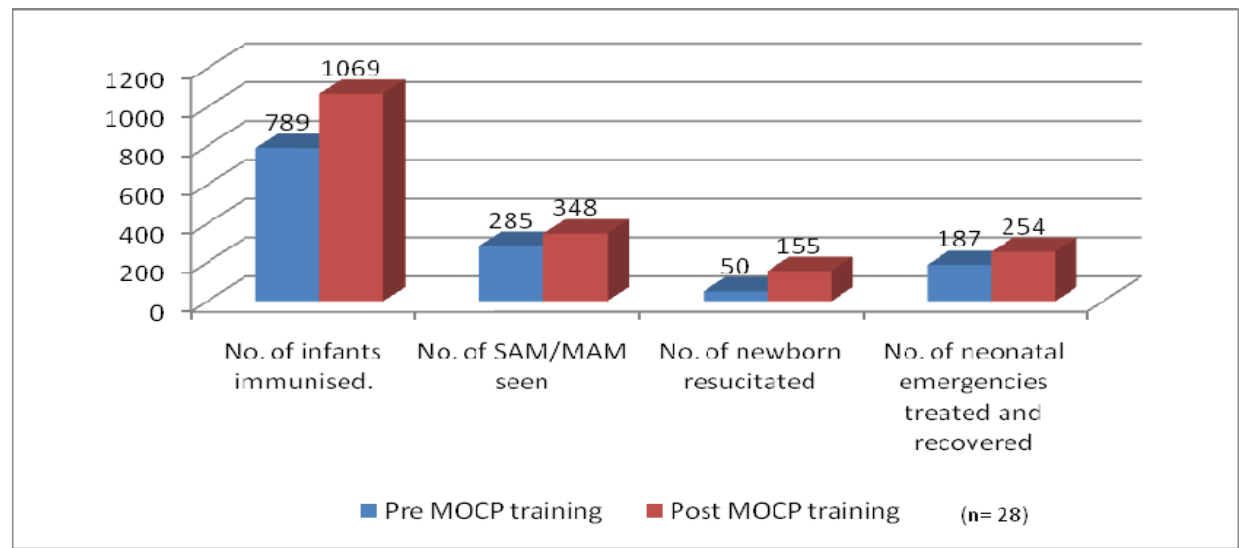

Figure 3. Management of Paediatric patients pre and post MOCP training

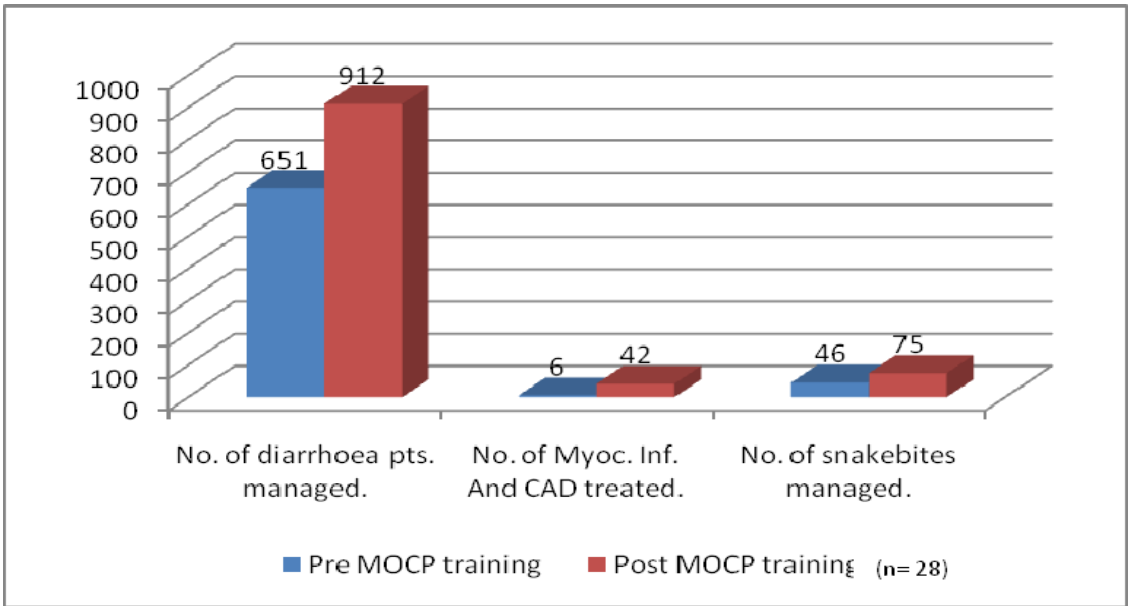

Figure 4. Disease management pre and post MOCP training

Figure 6 shows the number of medical emergencies handled, number of procedures performed such as insertion of vein flow, Ryle's tube insertion, intubation, etc and number of ECGs taken in patients pre and post MOCP training. From the figure it can be seen that number of medical emergencies managed and number of ECGs taken has drastically increased in post MOCP training than pre MOCP training.

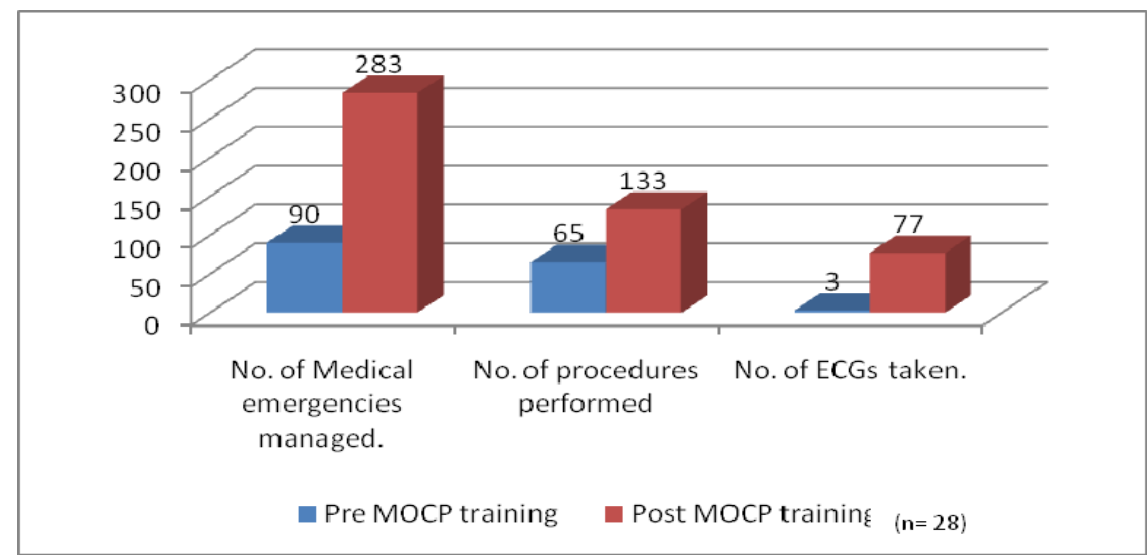

Figure 5. Procedures and management of medical emergencies pre and post MOCP training 
Figure 7 shows the number of OPD patients treated in 18 districts of Maharashtra. From the figure it can be seen that except for Parbhani and Bhandara, in all the other districts there has been a rise in number of OPD patients treated. Maximum number of OPD patients treated was reported in the district of Latur. This indicates the reduction of morbidity in those districts after receiving MOCP training.

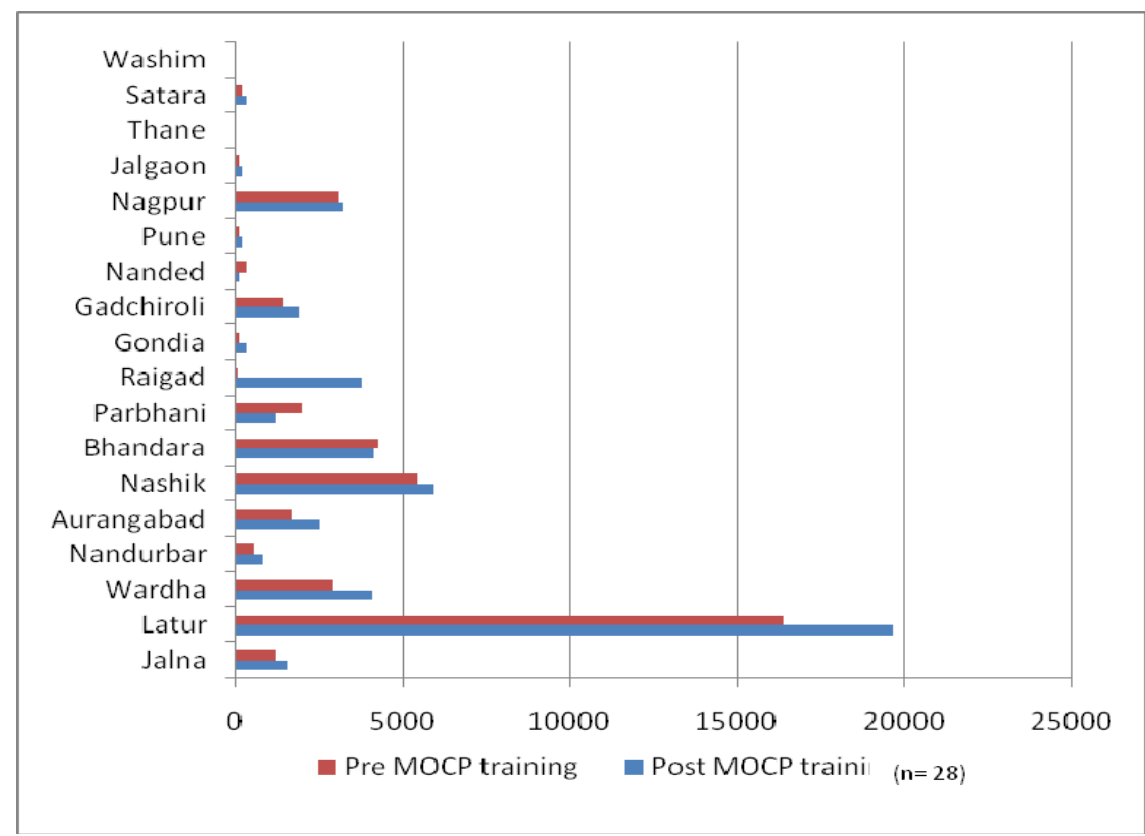

Figure 6. District wise number of OPD patients pre and post MOCP training

Figure 8 shows the number of IPD patients treated in 18 districts of Maharashtra. From the figure it can be seen that except for Parbhani and Bhandara, in all the other districts there has been a rise in number of IPD patients treated. Maximum number of IPD patients treated was reported in the district of Latur. This indicates the reduction of morbidity in those districts after receiving MOCP training.

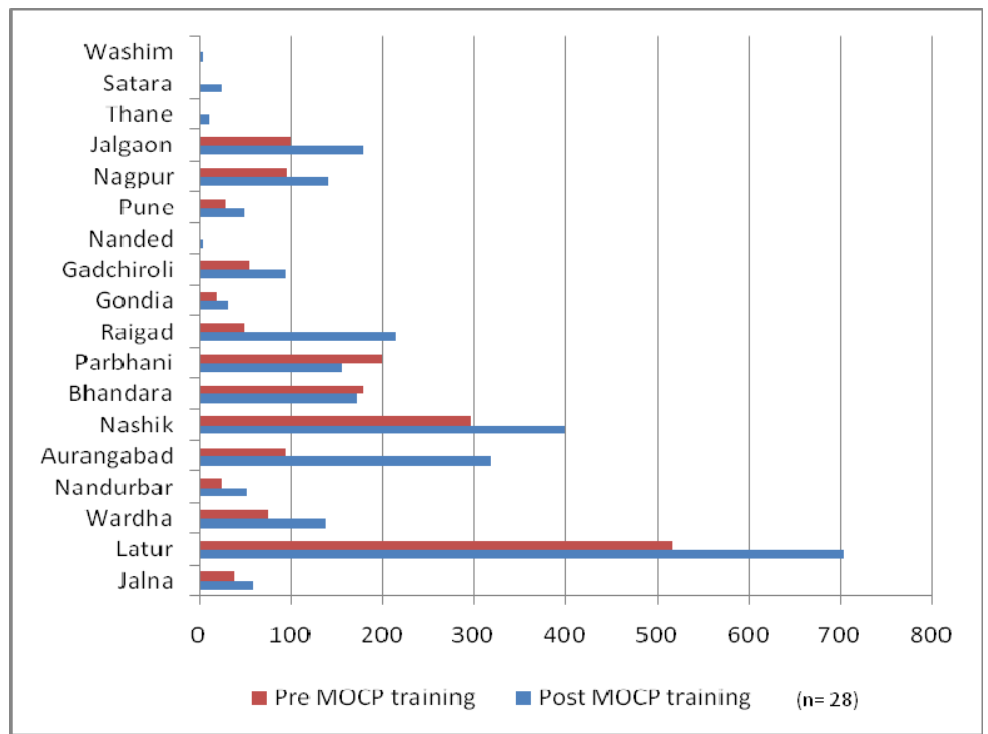

Figure 7. District wise number of IPD patients pre and post MOCP training 


\section{CONCLUSION}

MOCP course is thus a unique course in the country and it has helped to fill the shortage of Pediatricians and Physicians at Primary Health Centers in the State of Maharashtra. Improving the competence of the Medical officers working in the Public Health Departments all over is the need of the hour $1,2,3$. Through the MOCP course, doctors were trained resulting in skill enhancement..It also helped in patient management.They opined that they learnt the state of art treatment, There was an increase in the number of indoor and outdoor patients, increase in number of patients with malnutrition, snake bite, myocardial infarction, jaundice sepsis etc. treated and number of ECGs taken.Their confidence level was increased. Overall doctors and patients were both satisfied after the training.

\section{REFERENCES}

[1] Patricia Aparicio, et al., "Evaluation of Medical Students in the training of Paediatric Life Support. A Spanish Perspective", Journal of Emergency Primary Health Care, vol/issue: 1(3). Website: http://ro.ecu.edu.au/jephc/vol1/iss3/12.

[2] HN Kamiru, et al., "Effectiveness of a training program to increase the capacity of health care providers to provide HIV/AIDS care and treatment in Swaziland”, AIDS Care, vol/issue: 21(11), pp. 1463-1470, 2009.

[3] Satyavati Devi, "Short term training of Medical Officers in Mental Health”, Indian Journal of Psychiatry, vol/issue: 35(2), pp. 107-110, 1993 